\title{
Large Magnetocaloric Effect with a Wide Working Temperature Span in the Intermetallic Compound $\mathrm{Nd}_{4} \mathrm{PtMg}$
}

\author{
Devi Prasadh P S ${ }^{1}$ and B. K. Sarkar ${ }^{2}$ \\ ${ }^{1}$ Department of Physics, Dr.Mahalingam College of Engg.\& Tech., Pollachi, Coimbatore - 642 003, India. \\ ${ }^{2}$ Department of Physics, Galgotias University, Greater Noida - 201 308, India.
}

\begin{abstract}
The magnetic and magneto-caloric properties of intermetallic compound, $\mathrm{Nd}_{4} P t M g$ have investigated by magnetization and heat capacity measurements. The compound exists in prototype $G d_{4} R h I n$ cubic type structure. It has been observed that $\mathrm{Nd}_{4} \mathrm{PtMg}$ changes from paramagnetic to ferromagnetic state with a second order magnetic phase transition at Curie temperature of $T_{C} \sim 26 \mathrm{~K}$. The compound shows a large reversible magneto-caloric effect around $T_{C}$. In the varying magnetic field, $\mathrm{Nd}_{4} P t M g$ undergoes magnetic entropy change with a maximum value of $21.3 \mathrm{~J} / \mathrm{kg}-\mathrm{K}$.
\end{abstract}

Keywords: Magneto-caloric Effect, Phase transition, Curie temperature

\section{Introduction}

Recent research on magnetic refrigeration based on the magneto caloric effect (MCE) has fascinated much attention. Many are concentrating on MCE technology because of its higher energy efficiency and lower environmental hazard (eco-friendly) concern when compared to the conventional gas compression method. Magnetic materials with large magneto-caloric effect have been studied in a detailed fashion in both experimental and theoretical methods for the better understanding the properties of the materials [1-16]. MCE near to the room temperature is used for industrial refrigeration [17], which can reduce the greenhouse gases. So that only researchers are giving importance to search magnetic refrigerants at room temperature [18]. The rare earth (RE) intermetallic compounds of the type $\mathrm{RE}_{4} \mathrm{TX}(\mathrm{T}=$ transition metal; $\mathrm{X}=\mathrm{Mg})$, crystallize in the cubic $\mathrm{Gd}_{4} \mathrm{RhIn}$ - type structure have attracted much attention because of their fascinating magnetic \& magneto-caloric properties [19] and wide practical applications. When a magnetic field is applied to a magnetic material, a remarkable change will be induced in the material's polarization. The magnetic materials which have the first order magnetic and structural transition show large MCE. The decrease in the refrigeration efficiency of the intermetallic material is due to the thermal and magnetic hysteresis connected with the first order magnetic structural transition.The MCE is a magneto-thermodynamic phenomenon which displays as an isothermal magnetic entropy change or an adiabatic temperature change when the magnetic material is exposed to a varying magnetic field. It is necessary and important to find and investigate new materials with large MCE because for better applications the large values of isothermal magnetic entropy change and adiabatic temperature change are considered. Available first ordered phase transition materials are narrow. So, for better understanding of the magneto-caloric effect and searching for such materials with large MCE, we have studied the intermetallic compound, $\mathrm{Nd}_{4} \mathrm{PtMg}$. In this paper, magnetic properties and $\mathrm{MCE}$ in $\mathrm{Nd}_{4} \mathrm{PtMg}$ were discussed in detail.

\section{Experimental}

A high quality (99.9\%) sample of $\mathrm{Nd}, \mathrm{Pt}$ and $\mathrm{Mg}$ were synthesized by the induction melting method. They are in sealed Ta tubes in water cooled sample chamber. The stoichiometric amounts of $\mathrm{Nd}, \mathrm{Pt}$ and $\mathrm{Mg}$ were melted six times to ensure the homogeneity on a water cooled copper hearth. The total weight loss of the sample is in this step was approximately $0.5 \%$. Then the sample was annealed at $1180 \mathrm{~K}$ for 7 days in evacuated quartz tubes to improve the homogeneity. The sample was single phase as confirmed by $\mathrm{X}-$ ray powder diffraction (XRD) and Energy Dispersive X - ray Spectroscopy (EDXS) analyses. The magnetic measurements were done up to $7 \mathrm{~T}$ field with the help of Vibrating Sample Magnetometer (VSM). The specific heat was measured by the adiabatic calorimeter. The MCE can be measured directly or indirectly either by the calculated magnetization or the field dependence of the heat capacity. 


\section{Results And Discussion}

The temperature dependence of the zero field cooled (ZFC) and field cooled (FC) dc susceptibility ( $\square$ $=\mathrm{M} / \mathrm{H}$ ) under a low magnetic field of $0.1 \mathrm{~T}$ for $\mathrm{Nd}_{4} \mathrm{PtMg}$ is displayed in figure-1. It is observed that paramagnetic to ferromagnetic (PM-FM) transition occurs around the Curie temperature $\mathrm{T}_{\mathrm{C}} \sim 26 \mathrm{~K}$ and also it is observed that the compound exists in prototype $\mathrm{Gd}_{4} \mathrm{RhIn}$ cubic type structure. The thermal hysteresis cannot be observed between ZFC and FC $\square$-T curve on and over the Curie temperature $T_{c}$. In the same fashion it is observed that some of the rare earth inter-metallic materials, such as $\mathrm{RE}_{4} \mathrm{PtMg}$ [20], REAgAl [21] and so on, the thermo-magnetic irreversibility in the present $\mathrm{Nd}_{4} \mathrm{PtMg}$ is due to the domain wall pinning effects. The magnetic entropy change $\Delta \mathrm{S}_{\mathrm{M}}$ was calculated from the temperature and field dependence of the magnetization $\mathrm{M}(\mathrm{H}, \mathrm{T})$ using the Maxwell's thermodynamic relation.

$\Delta S_{M}(T, \Delta H)=\int_{0}^{H^{\text {rrusex }}}\left(\frac{\partial M(H, T)}{\partial T}\right)_{H} d H$

The resulting temperature dependence of $-\Delta \mathrm{S}_{\mathrm{M}}$ with various magnetic field changes for $\mathrm{Nd}_{4} \mathrm{PtMg}$ was evaluated. The maximum values of magnetic entropy change $-\Delta S_{M}{ }^{\text {Max }}$ is calculated to be $21.3 \mathrm{~J} / \mathrm{kg}-\mathrm{K}$. from the result of the $-\Delta S_{M}{ }^{M a x}$ we can conclude that the present study comes under the category of the large MCE materials. The relative cooling power (RCP) also can be calculated from $-\Delta S_{M}{ }^{\text {Max }}$. RCP is defined as the product of the maximum magnetic entropy change $-\Delta S_{M}{ }^{M a x}$ and full width at half maximum in the $-\Delta S_{M}(T)$ graph. Figure-2 shows the temperature variation of specific heat and a peak observed around $\mathrm{T}_{\mathrm{C}}$ confirms the signature of second order phase transition. From the figure -2 , it is observed that a neat $\Lambda$ shape is found near the critical temperature $T_{c}$ which is another typical sign of a second order phase transition. This data supplies the determination for $T_{c}=26.2 \mathrm{~K}$, which was regular with that well-known from $M(T)$ curve.

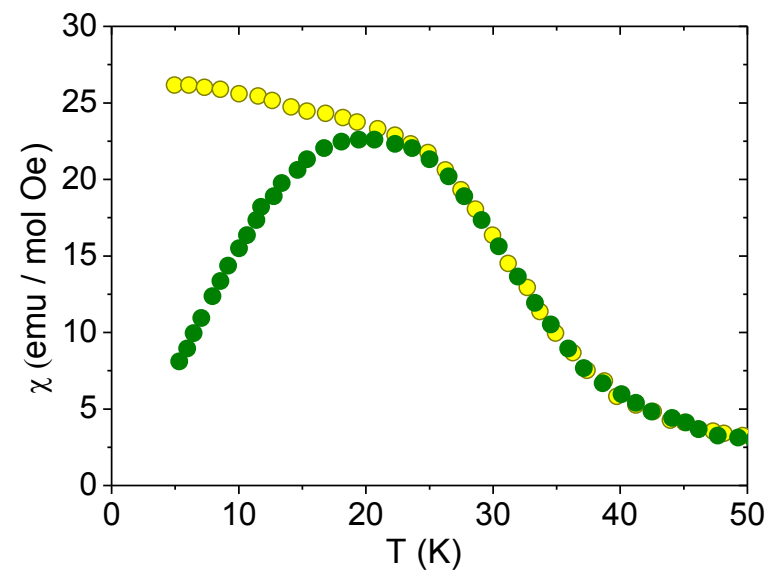

Fig.1. The temperature dependence of ZFC and FC susceptibility $(\chi)$ under a low field of $0.1 \mathrm{~T}$.

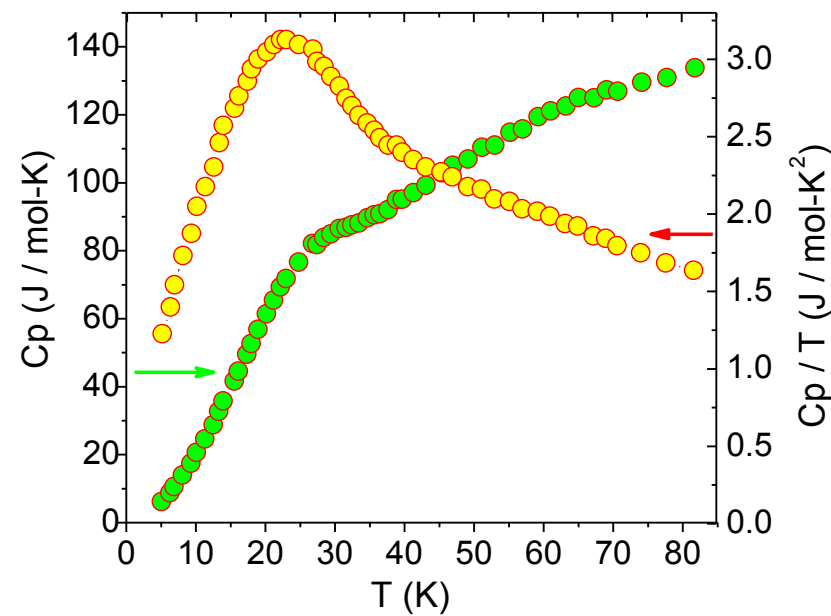

Fig.2. The temperature dependence of specific heat under zero magnetic field. 


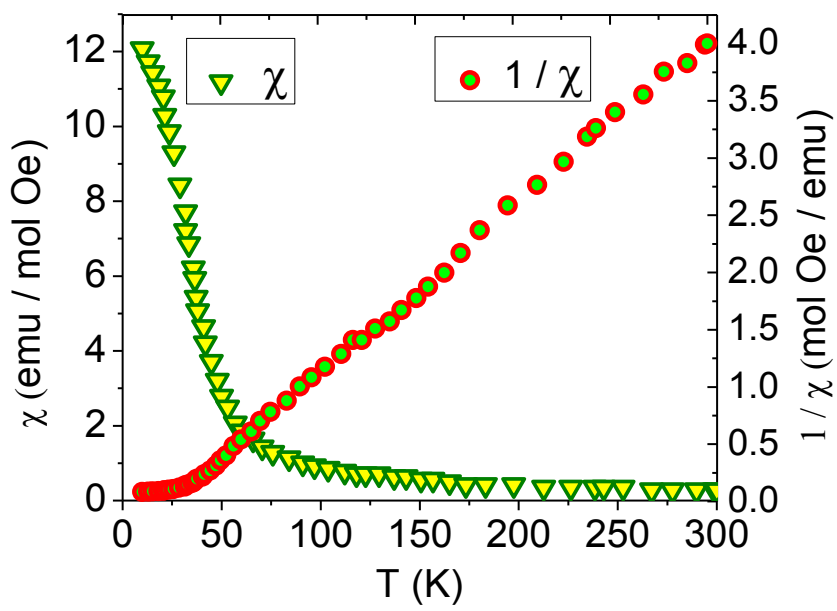

Fig.3. The temperature dependence of susceptibility.

Table1: The transition temperature $\left(\mathrm{T}_{\mathrm{M}}\right)$ and the maximum magnetic entropy change $\left(-\Delta \mathrm{S}_{\mathrm{M}}{ }^{\mathrm{Max}}\right)$ for $\mathrm{Nd}_{4} \mathrm{PtMg}$ and also for various large MCE materials.

\begin{tabular}{|l|l|l|l|}
\hline Material & $\mathrm{T}_{\mathrm{M}}(\mathrm{K})$ & $\left(-\Delta \mathrm{S}_{\mathrm{M}}^{\mathrm{Max}}\right) \mathrm{J} / \mathrm{kg}-\mathrm{K}$ & Reference \\
\hline $\mathrm{Er}_{4} \mathrm{PtMg}$ & 15 & 17.9 & 20 \\
\hline $\mathrm{HO}_{4} \mathrm{PtMg}$ & 28 & 13.4 & 20 \\
\hline $\mathrm{ErRu}_{2} \mathrm{Si}_{2}$ & 5.5 & 17.6 & 22 \\
\hline $\mathrm{GdCO}_{3} \mathrm{~B}_{2}$ & 54 & 9.4 & 23 \\
\hline $\mathrm{HoZn}$ & 72 & 12.1 & 24 \\
\hline $\mathrm{HoPd}$ & 10 & 11.3 & 11 \\
\hline $\mathrm{Er}$ & 13 & 12.0 & 25 \\
\hline $\mathrm{Nd}_{4} \mathrm{PtMg}$ & 26 & 21.3 & Present \\
\hline
\end{tabular}

The susceptibility and reciprocal susceptibility is shown in figure-3.1/ $\chi$ shows Curie - Weiss behavior above $100 \mathrm{~K}$. the value of the effective magnetic moment $\mu_{\text {eff }}$ is identified to be $12.2 \mu \mathrm{B} / \mathrm{RE}$. The related paramagnetic Curie temperature $\theta_{\mathrm{P}}$ was obtained. From the result it is concluded that the magnetic states is in long range ferromagnetic interaction.

\section{Conclusion}

In summary, single phased $\mathrm{Nd}_{4} \mathrm{PtMg}$ compound has been successfully synthesized and the magnetic and magnetocaloric properties have been investigated. From magnetic measurement, it has been observed that $\mathrm{Nd}_{4} \mathrm{PtMg}$ changes from paramagnetic to ferromagnetic state with a second order magnetic phase transition at Curie temperatures of $\mathrm{T}_{\mathrm{C}} \sim 26 \mathrm{~K}$ and there is no thermal hysteresis found at this temperature. The compound demonstrates a large reversible magneto-caloric effect around $\mathrm{T}_{\mathrm{C}}$. With magnetic field reversal, $\mathrm{Nd}_{4} \mathrm{PtMg}$ reveals a magnetic entropy change with a maximum value of $21.3 \mathrm{~J} / \mathrm{kg}$-K.The present work gives remarkable information to the researchers who are searching suitable materials which exhibit two or more successive magnetic phase transitions.

\section{Acknowledgements}

We are thankful to Dr. Mahalingam college of Engg. \& Tech., Coimbatore and Galgotias University, Greater Noida, for their support.

\section{References}

[1]. K.A. Gschneidner Jr., V.K. Pecharsky and A.O. Tsokol, Recent developments in magnetocaloric materials, Reports on Progress in Physics, 68, 2005, 1479 - 1539.

[2]. Shen B G, Hu FX, Dong QY and Sun JR, Magnetic properties and magnetocaloric effects in $\mathrm{NaZn}_{13}-\mathrm{type} \mathrm{La}(\mathrm{Fe}$, Al) ${ }_{13}$-based compounds, Chinese Physics B, 22 (1), 2013, 017502.

[3]. Pecharsky VK, Gschneidner Jr. KA andTsokol AO, Giant Magnetocaloric Effect in $\mathrm{Gd}_{5}\left(\mathrm{Si}_{2} \mathrm{Ge}_{2}\right)$, Physical Review Letters, 78,1997, 4494.

[4]. L. Li, O. Niehaus, M. Kersting and R. Pöttgen, Reversible table-like magnetocaloric effect in $\mathrm{Eu}_{4} \mathrm{PdMg}_{\mathrm{M}}$ over a very large temperature span, Applied Physics Letters, 104, 9, 2014, 092416 (1-4). 
[5]. Lingwei Li, Yasuhiro Nishimura, DexuanHuo, and ZhenghongQian, Giant magnetocaloric effect in antiferromagnetic borocarbide superconductor $\mathrm{RNi}_{2} \mathrm{~B}_{2} \mathrm{C}(\mathrm{R}=\mathrm{Dy}$, Ho, and Er) compounds, Journal of Applied Physics, 110, 2011, 083915.

[6]. R. Bjork, C.R.H. Bahl, A. Smith and N. Pryds, Review and comparison of magnet designs for magnetic refrigeration, International journal of refrigeration, 33, 2010, 437 - 448.

[7]. X.C. Zhong, J.X. Min, Z.W. liu, Z.G. Zheng, D.C. Zeng and V. Franco, Low hysteresis and large room temperature magnetocaloric effect of $\mathrm{Gd}_{5} \mathrm{Si}_{2.052 x} \mathrm{Ge}_{1.952 x} \mathrm{Ni}_{2 \times}$ (2x 5 0.08, 0.1) alloys, Journal of Applied Physics, 113, 2013, 17 A916.

[8]. Vitaliji K. Pecharsky and Karl A. Gschneidner Jr., Magnetocaloric effect and magnetic refrigeration, Journal of Magnetism and Magnetic Materials, 200,1999, 44 - 56.

[9]. Lingwei Li, DexuanHuo, ZhenghongQian and Katsuhiko Nishimura, A comparative study of the magnetic properties and magnetic entropy change in $R \mathrm{Co}_{2} \mathrm{~B}_{2}(R=\mathrm{Pr}, \mathrm{Nd}$ and $\mathrm{Gd})$ compounds, Journal of Physics, Conference Series 263, 2011, 012017 (1-8).

[10]. N.K. Singh, K.G. Suresh, A.K. Nigam, S.K. Malik, A.A.Coelho and S. Gama, Itinerant electron metamagnetism and magnetocaloric effect in $\mathrm{RCo}_{2}$-based Laves phase compounds, Journal of Magnetism and Magnetic Materials, 317 , 2007, $68-79$.

[11]. Mo Zha0-Jun, Shen Jun, GaoXin-Qiang, Liu Yao, Wu Jian-Feng, ShenBao-Gen and Sun Ji-Rong, Magnetic properties and magnetocaloric effects in HoPd intermetallic, Chinese Physics B, 24-3, 2015, 037503 (1-4).

[12]. V.K. Pecharsky and K.A. Gschneidner Jr., Magnetocaloric effect from indirect measurements: Magnetization and heat capacity, Journal of Applied Physics Letters, 86, 1999, 565.

[13]. B.G. Shen, J.R. Sun, F.X. Hu, H.W. Zhang and Z. H. Chen, Recent Progress in Exploring Magnetocaloric Materials, Advanced Materials, 21, 2009, 4545.

[14]. S. Nishigori, Y. Hirooka and M. Guillot, Spin-glass behavior in TbPdIn and DyPdIn, Journal of Magnetism and Magnetic Materials, 177, 1998, 137 - 138.

[15]. Gschneider Jr. KA, The magnetocaloric effect, magnetic refrigeration and ductile intermetallic compounds, Acta materialia, 57, 2009, $18-28$.

[16]. Hu F X, Shen B G, Sun J R and Zhang, X-X, Great magnetic entropy change in $\mathrm{La}(\mathrm{Fe}, \mathrm{M}) 13(\mathrm{M}=\mathrm{Si}, \mathrm{Al})$ with Co doping, Chinese Physics, 9, 2000, 550 - 553.

[17]. B.F. Yu, Q. Gao, B. Zhang, X.Z. Meng, and Z. Chen, Review on research of room temperature magnetic refrigeration, International journal of refrigeration, 26 (6), 2003, 622 - 636.

[18]. Hu F.X., Shen B.G., and J.R. Sun, Magnetic entropy change in $\mathrm{Ni}_{51.5} \mathrm{Mn}_{22.7} \mathrm{Ga}_{25.8} \mathrm{Ni}_{51.5} \mathrm{Mn}_{22.7} \mathrm{Ga}_{25.8}$ alloy, Applied Physics Letters, 76, 2000, 3460.

[19]. M.H. Phan, G.T. Woods, A. Chathurvedi, S. Stefanoski, G.S. Nolas and H. Srikanth, Long-range ferromagnetism and giant magnetocaloric effect in type III Eu8Ga16Ge30Eu8Ga16Ge30 clathrates, Applied Physics Letters, 93, 2008, 252505.

[20]. Lingwei Li, Oliver Niehaus, Marcel kersting and Rainer Pottgen, Magnetic properties and magnetocaloric effect in the rare earth-rich phases $R E_{4} \mathrm{PtMg}(R E=\mathrm{Ho}$ and Er), Intermetallics, 62, 2015, 17 - 21.

[21]. Zhang Y, Yang B and Wilde G, Magnetic properties and magnetocaloric effect in ternary REAgAl $(\mathrm{RE}=\mathrm{Er}$ and $\mathrm{Ho})$ intermetallic compounds, Journal of Alloys Compounds, 619, 2015, 12 - 15.

[22]. T. Samantha, I. Das, and S. Banarjee, Magnetocaloric effect in $\mathrm{Ho}_{5} \mathrm{Pd}_{2} \mathrm{Ho}_{5} \mathrm{Pd}_{2}$ : Evidence of large cooling power, Applied Physics Letters, 91, 2007, 152506.

[23]. L. Li, K. Nishimura, D. Huo, Z. Qian, T. Namiki, Critical behaviour of the RCo3B2 (R = Gd, Tb and Dy) compounds, Journal of Alloys Compounds, 572, 2013, 205 - 208.

[24]. Lingwei Li, Ye Yuan, Yikun Zhang, Rainer Pottgen, and ShengqiangZhau, Magnetic phase transitions and large magnetic entropy change with a wide temperature span in HoZn, Journal of Alloys Compounds, 643, 2015, 147 151.

[25]. Shen J, Zhao J.L., Hu F.X., Sun J.R. and Shen B.G., Order of magnetic transition and large magnetocaloric effect in $\mathrm{Er}_{3} \mathrm{Co}$, Chinese Physics B, 19, 2010, 047502. 